\title{
Singular which, mention-some, and variable scope uniqueness*
}

\author{
Aron Hirsch \\ The Hebrew University of Jerusalem \\ Bernhard Schwarz \\ McGill University
}

\begin{abstract}
Singular wh-questions carry a uniqueness presupposition. Dayal (1996) proposed that uniqueness is triggered by an answer operator (ANS), which occurs highest in the question LF, outside the question nucleus. We observe data which we take to show that uniqueness may be triggered at a low scope site, beneath operators which themselves are within the question nucleus. In response to these "low uniqueness" cases, we remove the uniqueness presupposition from ANS, and suggest re-localizing it to the $w h$ itself, which can reconstruct into the question nucleus to take narrow scope. This paves the way for a weakening of ANS previously suggested in Fox 2013 to accommodate mention-some questions.
\end{abstract}

Keywords: questions, uniqueness, singular which, mention-some

\section{Introduction}

Singular $w h$-questions carry a uniqueness presupposition (e.g. Higginbotham \& May 1981; Dayal 1996). The question in (1a) presupposes (1b): that exactly one letter is missing. What is the source of the presupposition?

a. Which letter is missing?

b. $\quad \exists$ !x [letter $(\mathrm{x}) \wedge \operatorname{missing}(\mathrm{x})]$

Dayal (1996) proposed that uniqueness comes from an answer operator, ANS, which occurs highest in the question LF, outside the question nucleus. ANS applies to the question's extension, a set of possible answers, and introduces a presupposition which requires in the case of singular questions that the set contain exactly one true member. We refer to Dayal's approach as globalist: uniqueness is sourced to an operator applying to the question as a whole.

* We thank audiences at SALT 29 (UCLA), the XPrag workshop Exhaustivity in Questions and Answers (Tübingen), and the McGill Semantics Research Group. We are particularly indebted to Dylan Bumford, Danny Fox, Roger Schwarzschild, and Yimei Xiang. All errors are, of course, our own. A.H. was supported in part by a postdoctoral fellowship from Social Sciences and Humanities Research Council of Canada (SSHRC). Both authors were supported in part by SSHRC Insight grants \#435-2019-0143, \#435-2018-0524, and \#435-2016-1448. 
There is another possibility, however. Uniqueness might come from an operator lower in the LF, within the question nucleus. In that case, each individual answer to the question would carry a uniqueness presupposition, which would project globally. We refer to this alternative hypothesis as localist. Our aim is to tease apart the two possibilities, building a case for the localist view.

Our argument will involve scope. We present data which we take to instantiate the configuration in (2). The uniqueness presupposition is introduced at a low scope site (indicated with $\exists$ !), beneath an operator (Op) which itself is within the question nucleus. If so, the uniqueness trigger must occur within the nucleus, as well. The configuration in (2) is out of the reach of the globalist view. Because the ANS operator must be highest in the question LF to be interpreted, it necessarily takes scope over all operators within the nucleus.

$$
[C P[T P \ldots \text { Op } \ldots \exists ! \ldots]]
$$

We build a composition where the source of uniqueness is the $w h$ item itself, which reconstructs from its surface position into the question nucleus. Op in our configuration in (2) will be above the launching site of wh-movement and, as such, reconstruction can yield uniqueness at an appropriately low site. Reconstruction of the wh-phrase was argued to be possible in Rullmann \& Beck 1998, and the idea that the wh might be the source of uniqueness was considered in Champollion, Ciardelli \& Roelofsen 2017 and Uegaki 2018, 2019.

By motivating a localist view, our results relieve ANS of the burden to derive uniqueness. Dayal's ANS couples the triggering of a uniqueness presupposition with deriving a strong notion of what counts as a complete answer to a question, one which requires every question to have a unique complete answer. That notion is too strong for mention-some questions, which allow for multiple complete answers. The localist view paves the way for a weaker semantics for ANS, considered in Fox 2013 to accommodate mention-some.

\section{Uniqueness from ANS}

As a point of departure, we spell out in detail Dayal's globalist theory deriving uniqueness from ANS. A skeletal LF for (1a) is provided in (3).

(3) [ANS [CP which letter $\lambda 1 \mathrm{C}\left[{ }_{T P} \mathrm{t}_{1}\right.$ is missing $\left.\left.]\right]\right]$

The CP denotes (the characteristic function for) a Hamblin set containing those propositions which result from assigning to the $w h$-phrase's trace position different values given by the wh's restrictor. Assuming that a singular NP denotes a property which holds only of atomic entities, the function in (4a) (at the evaluation world w) 
characterizes the set in (4b). To facilitate exposition, we will routinely conflate a question's extension with the set it characterizes.

$$
\begin{aligned}
\text { a. } & \llbracket \mathrm{CP} \rrbracket & =\lambda \mathrm{p} . \exists \mathrm{x}[\operatorname{letter}(\mathrm{x})(\mathrm{w}) \wedge \mathrm{p}=\operatorname{missing}(\mathrm{x})] \\
\mathrm{b} . & & \approx\{\operatorname{missing}(a), \operatorname{missing}(b), \ldots\}
\end{aligned}
$$

Dayal's ANS operator, defined in (5), applies to the Hamblin set, and introduces the presupposition that it contain a strongest, or maximally informative, true proposition, that is, a proposition that is true and entails any other proposition that is true. Any set contains at most one such maximally informative true member. If the presupposition is met, the structure headed by ANS denotes this member.

$$
\begin{array}{r}
\llbracket \mathrm{ANS} \rrbracket=\lambda \mathrm{Q}_{\mathrm{st}, \mathrm{t} \cdot} \lambda \mathrm{w}: \exists \mathrm{p}[\mathrm{p}(\mathrm{w}) \wedge \mathrm{p} \in \mathrm{Q} \wedge \forall \mathrm{q}[\mathrm{q}(\mathrm{w}) \wedge \mathrm{q} \in \mathrm{Q} \rightarrow \mathrm{p} \subseteq \mathrm{q}]] \\
. \mathrm{lp}[\mathrm{p}(\mathrm{w}) \wedge \mathrm{p} \in \mathrm{Q} \wedge \forall \mathrm{q}[\mathrm{q}(\mathrm{w}) \wedge \mathrm{q} \in \mathrm{Q} \rightarrow \mathrm{p} \subseteq \mathrm{q}]]
\end{array}
$$

The LF in (3), then, presupposes that (4) contain a maximally informative true member. Importantly, the propositions in (4) are all logically independent of one another. For a set of propositions that are not related by entailment, the existence of a maximally informative true member is tantamount to the existence of a unique true member, as shown in (6a) (for a world w). In turn, (6a) amounts to (6b) and, in this way, Dayal derives the target uniqueness presupposition.

$$
\begin{aligned}
& \text { a. } \exists ! \mathrm{p}[\mathrm{p}(\mathrm{w}) \wedge \mathrm{p} \in\{\operatorname{missing}(a), \operatorname{missing}(b), \ldots\}] \\
& \text { b. } \exists ! \mathrm{x}[\operatorname{letter}(\mathrm{x})(\mathrm{w}) \wedge \operatorname{missing}(\mathrm{x})(\mathrm{w})]
\end{aligned}
$$

At the same time, uniqueness presuppositions are absent with plural $w h$-questions and questions whose $w h$ lacks an overt restrictor (e.g. what). Dayal predicts that pattern, as well. Consider (7). Whereas which letter in (1a) ranges just over atomic entities, Dayal assumes that what ranges over both atomic entities - say individual letters — and the plural entities constituted by their mereological sums (Link 1983). As a result, (7) is associated with the Hamblin set in (8). Due to the distributivity of missing, which guarantees the equivalence of missing $(x+y)$ with missing $(x) \wedge$ missing $(y)$, this set is closed under conjunction.

$$
\begin{aligned}
& \text { What is missing? } \\
& \{\operatorname{missing}(a), \operatorname{missing}(b), \operatorname{missing}(a+b), \ldots\}
\end{aligned}
$$

For a Hamblin set closed under conjunction, the existence of a maximally informative true member is guaranteed so long as there are one or more true members. The presupposition that ANS triggers is therefore tantamount to (9a) (in a world w). Since (9a) amounts to (9b), a mere existence presupposition, that there is at least one missing letter, is correctly derived for (7). 
Although Dayal's system makes powerful predictions, we will argue that the globalist derivation does not always correctly predict a question's presupposition. The localist system we propose aims to preserve the virtues of Dayal's, while also accounting for data problematic for global triggering. Before turning to our argument, we note two challenges to Dayal's system from prior literature.

\subsection{Prior challenge 1: mention-some}

The first challenge, raised by Fox (2013), targets the proposed coupling of the presupposition with a characterization of answerhood. On Dayal's analysis, the uniqueness presupposition is a side effect of ANS's characterization of a question's complete true answer as the strongest true proposition in the Hamblin set. But, this notion of a complete answer is known not to be viable in general.

By Dayal's notion of answerhood, every question must have a unique complete true answer. That is appropriate for singular which-question like (1a), which can have exactly one true answer, and certain non-singular questions, which receive a "mention-all" interpretation, as is most natural for (7). If $a$ and $b$ are missing in w, a true answer to (7) is intuitively complete only if it conveys that both $a$ and $b$ are missing. Thus, the only answer that is judged complete is (10a). (10b) and (10c) are merely partial answers. This is captured by ANS, since the proposition it outputs, the strongest true answer in (8), will be missing $(a+b)$.

What is missing?
a. $\quad A$ and $b$ are missing.
b. $A$ is missing.
c. $\quad B$ is missing.
(complete at $w$ ) (partial at $w)$ (partial at $w)$

However, there are also "mention-some" questions, which allow for multiple complete answers in the same world. Mention-some readings emerge in modalized questions, such as (11). Intuitively, (11) can be interpreted as asking the responder to name just one place we can get gas. Suppose that the places to get gas in w are Texaco and Shell. The question, then, can be completely resolved equally well with either the answer in (11a) or the one in (11b).

(11) Where can we get gas?

a. We can get gas at Texaco.

(complete at $w$ )

b. We can get gas at Shell.

(complete at $w$ ) 
Dayal's ANS does not yield the required result. Consider the Hamblin set in (12). Assuming we do not get gas at multiple places at any accessible world, propositions formed from pluralities, like $\diamond$ (gas(Texaco+Shell)), will be false. The remaining propositions are all logically independent of one another and, as such, ANS's presupposition imposes a uniqueness requirement, just as it did in (1a). When the presupposition is met (which it is not in w), ANS will return the unique true element of (12) as the sole complete answer to the question.

$$
\{\diamond(\text { gas }(\text { Texaco })), \diamond(\text { gas }(\text { Shell })), \diamond(\text { gas }(\text { Texaco+Shell })), \ldots\}
$$

Another possibility is that a distributivity operator is inserted into the structure at a scope site above can. Since distributing Texaco+Shell over can yields the proposition $(\diamond(\operatorname{gas}($ Texaco $)) \wedge \diamond(\operatorname{gas}($ Shell $)))$, the Hamblin set is then (13), rather than (12). This set is closed under conjunction, and ANS would yield an existence presupposition and a mention-all reading, like in (7). The strongest true proposition in (13) is $(\diamond(\operatorname{gas}($ Texaco $)) \wedge \diamond(\operatorname{gas}($ Shell $)))$, and ANS would output that proposition, the one conveying all the places we can get gas. ${ }^{1}$

$$
\{\diamond(\text { gas }(\text { Texaco })), \diamond(\text { gas }(\text { Shell })), \diamond(\text { gas }(\text { Texaco })) \wedge \diamond(\text { gas }(\text { Shell })), \ldots\}
$$

Hence, Dayal's system predicts either uniqueness or mention-all for (11). The optionality between complete answers characteristic of mention-some is not derived. We return to the topic of answerhood and mention-some in Section 5.

\subsection{Prior challenge 2: projection}

Uegaki $(2018,2019)$ considers how the uniqueness presupposition projects when the question is embedded under a non-veridical predicate. He observes that uniqueness interacts with the predicate. The sentence in (14) globally presupposes not that there is exactly one missing letter, but that Max believes there is.

1 There are other derivations which converge. Spector (2008) proposed that $w h$-phrases can optionally range over quantifiers, instead of entities, and leave quantifier-type traces. Xiang (2016) raises, among other parses, a derivation for (11) with a quantifier-type trace below the modal, as in (i). Suppose the restrictor of where includes not only the Montague lift of the entities given by Texaco and Shell, but also disjunctive quantifiers such as Texaco or Shell. In the corresponding Hamblin answer, the disjunction takes scope beneath the modal, at the trace position $\left(\mathrm{t}_{2}\right)$. Xiang proposes that a covert operator, indicated here as EXH, can derive free choice strengthening of $\diamond($ gas(Texaco) $\vee$ $\operatorname{gas}($ Shell $))$ to $(\diamond($ gas $($ Texaco $)) \wedge \diamond($ gas $($ Shell $)))$ - closing the Hamblin set under conjunction. We will in general set aside parses with higher-order quantification. As far as we know, the existence of such parses would not impact our main arguments (but see fn. 7).

$$
\left[C P \text { where } \lambda 2 \mathrm{C}\left[\mathrm{EXH}\left[{ }_{T P} \text { can }\left[\mathrm{t}_{2 ; e s t, s t} \lambda 1\left[{ }_{\nu P} \text { we get gas (at) } \mathrm{t}_{1 ; e}\right]\right]\right]\right]\right]
$$


Max is certain about which letter is missing.

In a straightforward extension of Dayal's system to (14), the ANS operator would be interpreted relative to the matrix evaluation world w, so that (14) conveys that in every w' compatible with what Max is certain about in w, the actual answer to the embedded question in $\mathrm{w}$ is true in $\mathrm{w}^{\prime}$. In addition to deriving a problematic veridical assertion, ${ }^{2}$ with the world argument of ANS free, its presuppositions should project to the matrix level, unaffected by the modal. To solve the projection problem, Uegaki proposes that uniqueness is carried not by ANS, but by the individual Hamblin answers. Projection in (14) then unifies with (15), where the question is replaced with one of its answers, and the global presupposition is, again, that Max believes exactly one letter is missing. Our proposal will share with Uegaki's the feature that uniqueness is carried by each individual Hamblin answer.

Max is certain that the missing letter is $a$.

\section{Low uniqueness}

Adding to the challenges for ANS in (5), we now present the core data which we take to instantiate the scope configuration in (16). Since Op is within the question nucleus, and uniqueness is triggered beneath Op, the uniqueness trigger must be low, within the nucleus, as well. Since ANS operates on the question's Hamblin set, it cannot take scope within the nucleus, hence cannot scope below Op. ANS cannot be the source of low uniqueness, nor can other global operators. ${ }^{3}$

$$
[C P[T P \ldots \text { Op } \ldots \exists ! \ldots]]
$$

\subsection{High uniqueness is not obligatory}

In our data, Op is an existential quantifier expressed by a possibility modal. In (17), the infinitival to make a word ensures that could receives a teleological interpretation, and provides an overt domain restrictor, so that could existentially quantifies over worlds where the goal of making a word is achieved.

2 If ANS's world argument were bound by certain (co-bound with the world argument of the structure headed by ANS, here [ANS [which letter is missing]]), a trivial reading would result: that in every w' compatible with what Max is certain about in w, the answer to the question that is true in w' is true in w'. To derive the correct assertion, Uegaki (2018: Sec. 4.1) modifies the argument structure of ANS. Our main focus is matrix questions, so we will suppress his revision here.

3 Uegaki (2018) entertains the idea that an operator which is below ANS, but still outside the question nucleus, applies to the Hamblin set, and outputs a new set, which is like the input, but with each answer carrying a uniqueness presupposition. Low uniqueness counters that possibility: each answer must carry uniqueness by virtue of the presupposition being triggered within the nucleus. 
What is (17) predicted to presuppose? On the globalist account, uniqueness is triggered above the modal. (17) has the LF in (18), and the Hamblin set in (19), with each proposition modalized. As in (1a), the members of the Hamblin set are logically independent, and the presupposition triggered by ANS requires that exactly one of the answers be true in the evaluation world $\mathrm{w}$, as in (20a). This amounts to (20b): that there exists exactly one letter that could be added to form a word. We refer to (20b) as a high uniqueness presupposition.

[ANS [CP which letter could we add]]

$$
\{\diamond(\operatorname{add}(a)), \diamond(\operatorname{add}(b)), \ldots\}
$$

$$
\text { a. } \quad \exists ! \mathrm{p}[\mathrm{p}(\mathrm{w}) \wedge \mathrm{p} \in\{\diamond(\operatorname{add}(a)), \diamond(\operatorname{add}(b)), \ldots\}]
$$

b. $\quad \exists$ !x [letter $\left.(\mathrm{x})(\mathrm{w}) \wedge \diamond_{\mathrm{w}}(\operatorname{add}(\mathrm{x}))\right]$

(high uniqueness)

It seems that (17) can indeed be understood in this way. However, (17) does not pattern as though it obligatorily presupposes high uniqueness. Suppose, for example, that (17) occurs in the context in (21), which provides a word skeleton that sets the implicit argument for $a d d$ in (18). The skeleton fo $m$ contains a single blank that must be filled to create a word. In this context, the high uniqueness presupposition fails at the actual world. There are, in fact, two letters that could be added. We could fill in the blank with $a$ to create the word foam, or $r$ to create form. Correspondingly, the Hamblin set in (19) contains two true members $-\diamond(\operatorname{add}(a))$ and $\diamond(\operatorname{add}(r))-$ contrary to the presupposition. Yet, (17) still seems perfectly natural.

The skeleton is fo_m.

As confirmation, we observe, first, that (17) can felicitously receive an answer incompatible with the presupposition. Repeated as (A) in (22), (17) can be answered with the disjunction in (B), under a free choice interpretation. That is, (B) can convey that each of $a$ and $r$ is a possible addition to create a word.

A: Which letter could we add to make a word?

B: $A$ or $r$.

One might wonder whether the felicity of (B) is actually compatible with (17) presupposing (20), after all. Conceivably, it could be that the questioner in (A) does intend to presuppose high uniqueness, and that the responder offers (B) as a felicitous presupposition denial. However, this proposal depends on a satisfactory explanation for the contrast between (22) and the control in (23). 
A: Which letter is missing in this word?

$\mathrm{B}$ : \#A and $r$.

The response in (23B) is to be read with neutral prosody, parallel to the natural prosodic realization of (22B). With such a prosody, (23B) is infelicitous. That is as it should be, given that (23B) contradicts the uniqueness presupposition attested for (23A), that exactly one letter is missing. If (23B) cannot felicitously deny the uniqueness presupposition of (23A), it is unclear why (22B) would be able to serve as a presupposition denial either.

We can also change the example to further mitigate any confound from the possibility of presupposition denial. In (24), the questioner herself offers a follow-up which makes clear that she does not intend to presuppose high uniqueness, and that is felicitous, as well. For good measure, we can show independently that a questioner cannot in general contradict her own uniqueness presupposition: (25) is infelicitous, since the follow-up contradicts the attested presupposition that exactly one letter is missing. We conclude that (17) need not presuppose high uniqueness.

Which letter could we add to make a word? $A$ is one. Others?

\#Which letter is missing in this word? A is. Others?

\subsection{Diagnosing low uniqueness}

We observe that there is still a kind of uniqueness associated with (17). To bring out the effect, suppose that the context is modified as in (26), so that the word skeleton contains two blanks, instead of one. Assuming that the question is asking about letter tokens, it registers as sharply deviant in the revised context.

(The skeleton is $f_{-}-m$.)

\#Which letter (token) could we add to make a word?

Even if the question does not presuppose that there is exactly one letter we could add to make a word, it still presupposes that we could make a word by adding exactly one letter. Intuitively, the infelicity in (26) is due to the context establishing that more than one letter must be added.

As we will show in detail in the next section, the observed presupposition is expected if uniqueness is triggered locally, within the modal's scope. Since the triggered presupposition excludes the modal, it has the content in (27a) (at a world w): that we add exactly one letter. Assuming that the triggered presupposition projects existentially through the possibility modal, the global presupposition is (27b): that, at some accessible world where we make a word, we add exactly one letter. Comparing (27b) to the high presupposition in (20b), the relative scope of 
uniqueness and the modal are reversed. Whereas $\exists$ ! scopes over the modal in (20b), the modal takes wide scope over $\exists$ ! in (27b). Accordingly, we refer to (27b) as the low uniqueness presupposition.

$$
\begin{array}{llr}
\text { a. } & \exists ! \mathrm{x}[\operatorname{letter}(\mathrm{x})(\mathrm{w}) \wedge \operatorname{add}(\mathrm{x})(\mathrm{w})] & \text { (triggered) } \\
\text { b. } & \diamond_{\mathrm{w}} \exists ! \mathrm{x}[\operatorname{letter}(\mathrm{x}) \wedge \operatorname{add}(\mathrm{x})] & \text { (projected; low uniqueness) }
\end{array}
$$

Assuming that a word skeleton is invariant across accessible worlds, once the word skeleton is set, the number of letters we add is uniform across all worlds where we make a word. With $f o \_m$, we add exactly one letter in all word-worlds, and the possibility requirement in (27b) is satisfied (indeed, even a stronger necessity requirement would be satisfied). With $f_{-} m$, there are no word-worlds where we add exactly one letter, and (27b) fails. Low uniqueness makes the right cut between contexts where the modalized question is and is not felicitous.

The low uniqueness effect is not tied to the presence of root modality. This is illustrated by the contrast in (28). (28a) has a felicitous interpretation where could is read as epistemic and thus asks about letters that, in view of the available evidence, could be missing. Intuitively, (28a) is compatible with there being multiple epistemic possibilities for the missing letter, indicating that a high uniqueness presupposition is not obligatory. Still, low uniqueness is again detectable in (28b).

a. Which letter could be missing in fo_m?

b. \#Which letter could be missing in $f_{-} m$ ?

In sum, we conclude that the uniqueness presupposition that Dayal's globalist account derives for singular which-questions is not always attested. When absent, it is supplanted by a uniqueness presupposition that is locally triggered and projected. The source of uniqueness, then, must be some scopally mobile element within the question nucleus. But, what is the localist trigger?

\section{Uniqueness from which}

Wh-phrases surface high in the left periphery. Do they also have to take scope at this high site? Rullmann \& Beck (1998) study cases where a wh-phrase extracts from an intensional context, and observe that in such cases, the wh-phrase's restrictor can be interpreted as being evaluated in worlds quantified over by the intensional operator. The question in (29), for example, need not be understood as asking about actual unicorns. Instead, (29) allows for a more natural interpretation on which felicitous answers identify entities that are unicorns in Bill's belief-worlds.

(Bill thought he saw two unicorns, a green one and a blue one.) Which unicorn did Bill want to catch? 
Rullmann \& Beck conclude that a wh-phrase's restrictor takes scope under another operator in virtue of the whole $w h$-phrase taking narrow scope. If $w h$-phrases have mobile scope, which has the right profile to be the uniqueness trigger, and we present an analysis in that vein. We tie low scope uniqueness to low scope for which.

\subsection{Step 1: uniqueness in basic data}

Our lexical entry for which is stated in (30). Which composes with an entity, and two properties. Which is accordingly what Hackl (2000) calls a parameterized determiner. Its assertive component returns truth in case the individual given by the first input has the properties given by the other two inputs. The entry in addition features a definedness condition that encodes a presupposition: that there is exactly one individual that satisfies both input properties.

$$
\begin{aligned}
& \llbracket \text { which } \\
& =\lambda \mathrm{x} \cdot \lambda \mathrm{f}_{\mathrm{e}, \mathrm{st}} \cdot \lambda \mathrm{g}_{\mathrm{e}, \mathrm{st}} \cdot \lambda \mathrm{w}: \exists ! \mathrm{y}[\mathrm{f}(\mathrm{y})(\mathrm{w}) \wedge \mathrm{g}(\mathrm{y})(\mathrm{w})] \cdot \mathrm{f}(\mathrm{x})(\mathrm{w}) \wedge \mathrm{g}(\mathrm{x})(\mathrm{w})
\end{aligned}
$$

The basic question in (31a) is derived as in (31b). A covert question forming operator, ?, originates as the first argument of which, and moves to take widest scope, leaving a type e trace. Which $(t)$ letter also moves to a peripheral position in the narrow syntax, but can reconstruct into the question nucleus at LF. For concreteness, we show reconstruction to the specifier of TP. The restrictor and scope arguments for which effectively correspond to letter and is missing, respectively.

a. Which letter is missing?

b. $\quad\left[C P\right.$ ? $\lambda 1\left[T P\left[D P\left[\right.\right.\right.$ which $\left.\mathrm{t}_{1}\right]$ letter $] \lambda 2\left[{ }_{v P} \mathrm{t}_{2}\right.$ is missing $\left.\left.]\right]\right]$

At the TP-level, which triggers the presupposition that there is exactly one entity that satisfies both letter and is missing, as underlined in (32). This is precisely the target presupposition that exactly one letter is missing. Now, the question remains: how does the presupposition project?

$$
\begin{aligned}
& \llbracket \mathrm{TP} \rrbracket^{g}=\llbracket \text { which } \rrbracket(\mathrm{g}(1))(\llbracket \operatorname{letter} \rrbracket)(\llbracket \text { is missing } \rrbracket)= \\
& \lambda \mathrm{w}: \exists ! \mathrm{y}[\operatorname{letter}(\mathrm{y})(\mathrm{w}) \wedge \operatorname{miss}(\mathrm{y})(\mathrm{w})] . \operatorname{letter}(\mathrm{g}(1))(\mathrm{w}) \wedge \operatorname{miss}(\mathrm{g}(1))(\mathrm{w})
\end{aligned}
$$

The TP asserts that the entity given by the trace of ? is a missing letter. ? has the denotation in (33), and binds its trace to output as the denotation of the CP the function in (34), characterizing the Hamblin set in (35). Each answer carries the presupposition that exactly one letter is missing, and asserts that some particular entity is a missing letter, with that entity differing between answers.

$$
\llbracket ? \rrbracket=\lambda \mathrm{f}_{\mathrm{e}, \mathrm{st}} \cdot \lambda \mathrm{p}_{\mathrm{st}} \cdot \exists \mathrm{x}[\mathrm{p}=\mathrm{f}(\mathrm{x})]
$$




$$
\begin{aligned}
& \lambda \text { p. } \exists \mathrm{x}[\mathrm{p}=\lambda \mathrm{w}: \exists ! \mathrm{y}[\operatorname{letter}(\mathrm{y})(\mathrm{w}) \wedge \operatorname{miss}(\mathrm{y})(\mathrm{w})] . \operatorname{letter}(\mathrm{x})(\mathrm{w}) \wedge \operatorname{miss}(\mathrm{x})(\mathrm{w})] \\
& \left\{\begin{array}{l}
\lambda \mathrm{w}: \exists ! \mathrm{y}[\operatorname{letter}(\mathrm{y})(\mathrm{w}) \wedge \operatorname{miss}(\mathrm{y})(\mathrm{w})] \cdot \operatorname{letter}(a)(\mathrm{w}) \wedge \operatorname{miss}(a)(\mathrm{w}), \\
\lambda \mathrm{w}: \exists ! \mathrm{y}[\operatorname{letter}(\mathrm{y})(\mathrm{w}) \wedge \operatorname{miss}(\mathrm{y})(\mathrm{w})] \cdot \operatorname{letter}(b)(\mathrm{w}) \wedge \operatorname{miss}(b)(\mathrm{w}), \\
\ldots
\end{array}\right\}
\end{aligned}
$$

We can show independently that a presupposition carried by each answer in a Hamblin set projects to become a presupposition of the question as a whole. For example, due to the definite description in the question nucleus, each answer to (36) will presuppose that France has a unique king, and this presupposition is intuited to be associated with the question. Given the Hamblin denotation in (34), we therefore arrive at the intended prediction: that (31) globally presupposes uniqueness.

(36) Who invited the king of France?

For basic data, the localist approach predicts the same overall presupposition as a globalist approach would - but through a different compositional path. While ANS would trigger the presupposition by operating on the Hamblin set, which triggers it within each Hamblin answer, and it projects from there.

\subsection{Step 2: low uniqueness = low scope which}

The two approaches come apart when an additional scope operator occurs in the question nucleus, as in our test data, the core case repeated in (37).

Which letter could we add to make a word?

While ANS introduced uniqueness at a fixed scope site above the modal, the whphrase can reconstruct to different positions in the question nucleus, leading to observed scope variability. We assume that when which letter moves in the narrow syntax it makes an intermediate landing (at least) at the edge of the local $v \mathrm{P}$ before raising to spec-TP and, finally, to its surface position in the periphery. In turn, which letter can reconstruct to spec-TP, above could, or to the edge of the $v \mathrm{P}$, below could. The two options are sketched in (38) and (39), respectively.

\section{LF1: which $>$ could}

$\left[C P ? \lambda 1\left[T P\left[D P\right.\right.\right.$ which $\mathrm{t}_{1}$ letter $] \lambda 3\left[T P\right.$ could $\left[{ }_{v P} \mathrm{t}_{3} \lambda 2\left[{ }_{v P}\right.\right.$ we add $\left.\left.\left.\left.\mathrm{t}_{2}\right]\right]\right]\right]$

\section{LF2: could $>$ which \\ $\left[C P\right.$ ? $\lambda 1\left[T P\right.$ could $\left[{ }_{v P}\left[D P\right.\right.$ which $\mathrm{t}_{1}$ letter $] \lambda 2\left[{ }_{v P}\right.$ we add $\left.\left.\left.\left.\mathrm{t}_{2}\right]\right]\right]\right]$}

LF1 yields the same high uniqueness reading derived with ANS, where it is globally presupposed that there is exactly one letter that we could add to make a word. It is 
the second parse, LF2, that furnishes a new reading: low uniqueness.

The $v \mathrm{P}$ structure in LF2 carries the presupposition in (40a). Assuming, again, that the presupposition projects existentially through the possibility modal, the TP presupposes (40b): that we could make a word by adding exactly one letter. That presupposition is, in turn, carried by each proposition in the Hamblin set that the $\mathrm{CP}$ expresses, as shown in (41), and projects globally. Because (40b) is precisely the low uniqueness presupposition that we identified for (37) in the last section, the analysis achieves empirical adequacy.

$$
\begin{aligned}
& \text { a. } \quad \exists \text { !y [letter }(\mathrm{y})(\mathrm{w}) \wedge \operatorname{add}(\mathrm{y})(\mathrm{w})] \quad \text { (triggered at } v P) \\
& \text { b. } \left.\diamond_{\mathrm{w}} \exists \text { !y [letter }(\mathrm{y}) \wedge \operatorname{add}(\mathrm{y})\right] \quad \text { (projected to } T P \text { ) } \\
& \left\{\begin{array}{l}
\lambda \mathrm{w}: \diamond_{\mathrm{w}} \exists ! \mathrm{y}[\operatorname{letter}(\mathrm{y}) \wedge \operatorname{add}(\mathrm{y})] \cdot \diamond_{\mathrm{w}}(\operatorname{letter}(a) \wedge \operatorname{add}(a)), \\
\lambda_{\mathrm{w}}: \diamond_{\mathrm{w}} \exists ! \mathrm{y}[\operatorname{letter}(\mathrm{y}) \wedge \operatorname{add}(\mathrm{y})] \cdot \diamond_{\mathrm{w}}(\operatorname{letter}(b) \wedge \operatorname{add}(b)), \\
\ldots
\end{array}\right\}
\end{aligned}
$$

\subsection{Comparison with Dayal}

The lexical entry for which in (30) requires a small update to generalize beyond data with a singular NP to data with a plural, as in (42). As discussed in Section 2 , non-singular questions do not presuppose uniqueness, but rather carry a simple existence presupposition, here that some letter is missing.

\section{Which letters are missing?}

With which defined as in (30), (42) is predicted to presuppose that there is exactly one entity which satisfies both letters and is missing. If letter holds of atomic letters, the plural letters would hold of both atomic letters, and plural entities with letters as atomic parts. If there are two letters missing, $a$ and $b$, the entities that satisfy letters include $a, b$, and the plurality $a+b$, all of which would (distributively) satisfy is missing, as well. Accordingly, while (42) intuitively allows for multiple missing letters, the presupposition of which fails in that case.

We can address this problem by adapting Sharvy's (1980) classic semantics for singular and plural definite descriptions. That is, in our entry for which in (30) above, we can revise the presupposition as in (43). Instead of demanding the existence of a unique entity that has the two properties, the revised condition merely requires that there by a maximal entity that has those properties, that is, an entity which has those properties and which contains all other entities that do.

$$
\begin{aligned}
& \llbracket \text { which } \\
& \begin{aligned}
=\lambda \mathrm{x} \cdot \lambda \mathrm{f}_{\text {est }} \cdot \lambda \mathrm{g}_{\text {est }} \cdot \lambda \mathrm{w}: & \exists \mathrm{y}[\mathrm{f}(\mathrm{y})(\mathrm{w}) \wedge \mathrm{g}(\mathrm{y})(\mathrm{w}) \wedge \forall \mathrm{z}[\mathrm{f}(\mathrm{z})(\mathrm{w}) \wedge \mathrm{g}(\mathrm{z})(\mathrm{w}) \rightarrow \mathrm{z} \leq \mathrm{y}]] \\
& . \mathrm{f}(\mathrm{x})(\mathrm{w}) \wedge \mathrm{g}(\mathrm{x})(\mathrm{w})
\end{aligned}
\end{aligned}
$$


For cases where the conjunction of the two properties can only hold of atomic entities, as guaranteed by singular marking on letter in our initial examples, the revised condition replicates the intended uniqueness presupposition. At the same time, in cases where the conjunction of the two properties is closed under sum formation, as in the plural example (42), the revised condition has the effect of merely deriving a presupposition of existence. If the missing letters are $a$ and $b$, the maximal entity which has the properties letters and is missing is the plurality $a+b$, and the revised presupposition is satisfied.

Our account, then, mirror's Dayal's globalist account in invoking maximality in the presupposition associated with singular and plural which questions. On the globalist account, maximality is based on an ordering of propositions in terms of logical strength. In our account, the ordering is the mereological part-whole relation between entities. Our system thus preserves the advantages of Dayal's, predicting uniqueness in singular which questions, and mere existence in non-singular data. By localizing maximality in which, we furthermore allow for maximality to be introduced at different scopes sites, as required for low uniqueness.

Our analysis for which would extend to what, at least when it occurs with an overt restrictor, as uniqueness effects again arise (e.g. What letter is missing?). In general, the null hypothesis is that the denotation for which is common to all wh-items, each encoding a maximality presupposition. When an overt restrictor is not present, a covert non-singular NP would furnish the first property argument.

\section{Consequences for answerhood}

We now consider consequences of our results for the lexical semantics of ANS and, relatedly, the notion of what counts as a complete answer to a question. If present in the structure, ANS must not trigger uniqueness. Fox (2013) proposes that ANS regulates whether a question receives a mention-all or mention-some interpretation. He offers a different notion of answerhood from Dayal's - one which allows some questions to have multiple complete answers - and weakens ANS in kind. One statement of ANS which Fox considers revises the operator in such a way that it does no longer trigger uniqueness. While Fox took failure to derive uniqueness to be a problem for the analysis, our results support such a formulation. Combining our system with Fox's ANS yields correct predictions about the distribution of mention-some readings with singular which questions.

\subsection{Weakening ANS}

As discussed in Section 2, Dayal took every licit question to have a unique complete answer, corresponding to the Hamblin set's strongest true element. What we refer to 
now as $\mathrm{ANS}_{D}$ presupposed that such an element exists, and returned that element. Fox (2013) considers a weaker formulation of ANS, provided in (44).

$$
\begin{aligned}
\llbracket \mathrm{ANS}_{F} \rrbracket=\lambda \mathrm{Q} . \lambda \mathrm{w}: & \exists \mathrm{p}\left[\mathrm{p}(\mathrm{w}) \wedge \mathrm{p} \in \mathrm{Q} \wedge \neg \exists \mathrm{p}^{\prime} \in \mathrm{Q}^{\prime}\left[\mathrm{p}^{\prime}(\mathrm{w}) \wedge \mathrm{p}^{\prime} \in \mathrm{Q} \wedge \mathrm{p}^{\prime} \subset \mathrm{p}\right]\right. \\
. & \left\{\mathrm{p}: \mathrm{p}(\mathrm{w}) \wedge \mathrm{p} \in \mathrm{Q} \wedge \neg \exists \mathrm{p}^{\prime} \in \mathrm{Q}\left[\mathrm{p}^{\prime}(\mathrm{w}) \wedge \mathrm{p}^{\prime} \in \mathrm{Q} \wedge \mathrm{p}^{\prime} \subset \mathrm{p}\right]\right\}
\end{aligned}
$$

$\mathrm{ANS}_{F}$ presupposes that the Hamblin set contain one or more propositions which are true and not entailed by an other true proposition. $\mathrm{ANS}_{F}$ returns the set of all such propositions, and any element of the ANS-set counts as a complete answer to the question. Depending on the logical make-up of the Hamblin set, the ANS-set may have necessarily one member, or allow for multiple members. In the latter case, a mention-some reading results.

For a Hamblin set closed under conjunction, $\mathrm{ANS}_{F}$ derives a mention-all reading, just as $\mathrm{ANS}_{D}$ did. Consider the question in (45), along with its classical Hamblin set in (46), which repeat (7) and (8). With the set closed under conjunction, if there is any true answer, there is necessarily a unique strongest true answer. That answer will also be the only true answer that is not entailed by any other true answer. Whereas $\mathrm{ANS}_{D}$ returned the strongest true answer itself, $\mathrm{ANS}_{F}$ will return the singleton set containing it. If, at w, $a$ and $b$ are missing, $\operatorname{ANS}_{F}$ will output the set in (47).

$$
\begin{aligned}
& \text { What is missing? } \\
& \{\operatorname{missing}(a), \operatorname{missing}(b), \operatorname{missing}(a+b), \ldots\} \\
& \llbracket \operatorname{ANS}_{F} \rrbracket((46))(\mathrm{w})=\{\operatorname{missing}(a+b)\}
\end{aligned}
$$

The move to $\mathrm{ANS}_{F}$ has crucial consequences when the Hamblin set is not closed under conjunction, and contains mutually compatible propositions. This is the case for (48) with the Hamblin set in (49), which repeat (11) and (12). Assuming as before that we will only get gas at a single place, and that Texaco and Shell are available at $\mathrm{w}$, the first two propositions in (49) are both true. Being logically independent, they are, moreover, both true propositions not entailed by any other true proposition. While the maximality presupposition of $\mathrm{ANS}_{D}$ would fail, the weaker presupposition of $\mathrm{ANS}_{F}$ is satisfied, and $\mathrm{ANS}_{F}$ returns the set of both propositions in (50), either of which constitutes a complete answer to the question. With multiple complete answers, (48) is correctly predicted to pattern as mention-some.

$$
\begin{aligned}
& \text { Where can we get gas? } \\
& \{\diamond(\operatorname{gas}(\text { Texaco })), \diamond(\operatorname{gas}(\text { Shell })), \diamond(\operatorname{gas}(\text { Texaco }+ \text { Shell })), \ldots\} \\
& \llbracket \mathrm{ANS}_{F} \rrbracket((49))(\mathrm{w})=\{\diamond(\operatorname{gas}(\text { Texaco }), \diamond(\operatorname{gas}(\text { Shell }))\}
\end{aligned}
$$

In this way, Fox's proposal derives both mention-all and mention-some readings, 
and makes predictions about their respective distribution. It predicts, for instance, that a non-modalized question (with a non-singular wh-phrase), like (45), should only permit a mention-all reading. George (2011) noted that this is the case, and Fox extended his observations with the minimal pair in (51). (51b), but not (51a), can ask the responder to name one place that now has gas.

(A truck delivered gas to five local gas stations.)

a. Where did he deliver gas?

$(\mathrm{MA}, * \mathrm{MS})$

b. Where can we get gas?

(MA, MS)

Quantitative data in Xiang \& Cremers (2017) further substantiate a contrast in the availability of mention-some between non-modalized and modalized questions, and Xiang (2016) provides additional arguments that the contrast between mention-all and mention-some readings should be regulated by a grammatical operator.

\subsection{Fox's dilemma}

Now, what does the re-formulated ANS predict for basic singular which questions? In combination with a classical analysis, where which does not itself encode uniqueness, $\mathrm{ANS}_{F}$ predicts no uniqueness at all. Consider (52) and its classical Hamblin set (53), repeating (3) and (4b) from Section 2. Like (49), (53) is not closed under conjunction, and contains multiple mutually compatible elements. Assuming the word is ferm, with $o$ and $r$ missing, there are two true propositions at w: $\operatorname{miss}(o)$ and $\operatorname{miss}(r)$. These are logically independent of one another, so both are true and not entailed by any other true proposition. The presupposition of $\mathrm{ANS}_{F}$ is met, and the doubleton containing both is output in (54).

(52) Which letter is missing?

(53) $\quad\{\operatorname{missing}(a), \operatorname{missing}(b), \ldots\}$

$$
\llbracket \mathrm{ANS}_{F} \rrbracket((53))(\mathrm{w})=\{\operatorname{missing}(o), \operatorname{missing}(r)\}
$$

In precisely the sort of case where (52) should suffer from failure of its uniqueness presupposition, the presupposition of $\mathrm{ANS}_{F}$ is met, and the question is predicted to pattern as mention-some, answerable with either proposition in (54).

$\mathrm{ANS}_{D}$ predicted uniqueness in (52), but also wrongly predicted uniqueness (or mention-all) for (48). ANS $_{F}$ derives mention-some in both, wrongly for (52). Accepting the premise that ANS should be the source of uniqueness, Fox (2013, 2018) and Xiang (2016) suggest further modifications to ANS to derive uniqueness in (52), while still predicting mention-some in (48). Our analysis, on the other hand, denies the premise. By re-sourcing uniqueness away from $\mathrm{ANS}, \mathrm{ANS}_{F}$ can be 
maintained, and uniqueness still derived in (52).

In Section 4, we replaced the Hamblin set in (53) with (55) which, most crucially, adds to each proposition in the set a uniqueness presupposition from which. With the presupposition projected globally, ${ }^{4}$ while the Hamblin set is still not closed under conjunction, the propositions it contains are rendered mutually exclusive.

$$
\left\{\begin{array}{l}
\lambda \mathrm{w}: \exists ! \mathrm{y}[\operatorname{letter}(\mathrm{y})(\mathrm{w}) \wedge \operatorname{miss}(\mathrm{y})(\mathrm{w})] . \operatorname{letter}(a)(\mathrm{w}) \wedge \operatorname{miss}(a)(\mathrm{w}), \\
\lambda \mathrm{w}: \exists ! \mathrm{y}[\operatorname{letter}(\mathrm{y})(\mathrm{w}) \wedge \operatorname{miss}(\mathrm{y})(\mathrm{w})] . \operatorname{letter}(b)(\mathrm{w}) \wedge \operatorname{miss}(b)(\mathrm{w}), \\
\ldots
\end{array}\right\}
$$

With two letters missing in ferm, the uniqueness presupposition from which fails, ruling out the question. By contrast, if there is just a single missing letter, the uniqueness presupposition is satisfied, and there is just one true proposition in the Hamblin set. That proposition is trivially not entailed by any other true proposition, so the presupposition of $\mathrm{ANS}_{F}$ is satisfied in kind. $\mathrm{ANS}_{F}$ returns the singleton set containing the true proposition, as in (56), if the word is form.

$$
\begin{aligned}
& \llbracket \operatorname{ANS}_{F} \rrbracket((55))(\mathrm{w}) \\
& =\{\lambda \mathrm{w}: \exists ! \mathrm{y}[\operatorname{letter}(\mathrm{y})(\mathrm{w}) \wedge \operatorname{miss}(\mathrm{y})(\mathrm{w})] . \operatorname{letter}(r)(\mathrm{w}) \wedge \operatorname{miss}(r)(\mathrm{w})\}
\end{aligned}
$$

Hence, the prediction that singular which questions should pattern as mention-some is avoided. In contexts where the question is permissible according to its uniqueness presupposition, there is one true Hamblin answer, and $\mathrm{ANS}_{F}$ returns the singleton set thereof, identifying that answer as the sole complete true one. ${ }^{5}$

\subsection{Where mention-some is observed}

So far, we have shown that combining $\mathrm{ANS}_{F}$ with a separate uniqueness trigger in which avoids over-generating mention-some in basic singular which-questions. At the same time, with the identification of low uniqueness comes a further prediction, that there should be singular which-questions which actually do allow for mention-

4 The adoption of $\mathrm{ANS}_{F}$ has a further welcome effect within the context of our analysis: it accounts for the observation, exploited in Section 4, that a presupposition that is carried by all Hamblin answers projects as a presupposition of the question as a whole. This is because in virtue of demanding that one or more Hamblin answers be true, the presupposition triggered by $\mathrm{ANS}_{F}$ also demands that the presupposition of one or more Hamblin answers be met.

5 Introducing a maximality presupposition into where would not affect mention-some in (48). Where would be parsed with a covert non-singular NP $(\approx$ what places $)$ and, as such, maximality would introduce a mere existence presupposition. Scoping what NP over can would yield a presupposition that there is some place we can get gas. Scope under can would yield a triggered presupposition that we get gas somewhere, projecting that we can get gas somewhere (equivalent to the high presupposition, if the NP is interpreted relative to the evaluation world). Either way, the presupposition is compatible with there being multiple places we can get gas, allowing for mention-some. 
some: modalized questions on their low uniqueness reading. Repeating (37) and (41) from Section 4, our low uniqueness prototype is shown again in (57), along with the Hamblin set for the LF with which taking scope under could, in (58).

$$
\text { Which letter could we add to make a word? }
$$

$$
\left\{\begin{array}{l}
\lambda_{\mathrm{w}}: \diamond_{\mathrm{w}} \exists ! \mathrm{y}[\operatorname{letter}(\mathrm{y}) \wedge \operatorname{add}(\mathrm{y})] \cdot \diamond_{\mathrm{w}}(\operatorname{letter}(a) \wedge \operatorname{add}(a)), \\
\lambda_{\mathrm{w}}: \diamond_{\mathrm{w}} \exists ! \mathrm{y}[\operatorname{letter}(\mathrm{y}) \wedge \operatorname{add}(\mathrm{y})] \cdot \diamond_{\mathrm{w}}(\operatorname{letter}(b) \wedge \operatorname{add}(b)), \\
\ldots
\end{array}\right\}
$$

While the uniqueness presupposition in the basic case in (55) renders the elements of the Hamblin set mutually exclusive, the projected low uniqueness presupposition does not. It is presupposed that we could make a word by adding exactly one letter. That is compatible with us being able to add $a$ and us being able to add $b$, and so forth. Any of the propositions in (58) could be true at once.

With the familiar skeleton, $f o \_m$, where we could make a word by adding $a$ or $r$, the projected uniqueness presupposition is met, and there are two true propositions in the Hamblin set. Since those are logically independent of one another, neither is entailed by another true element of the Hamblin set. Accordingly, the presupposition of $\mathrm{ANS}_{F}$ is met, and $\mathrm{ANS}_{F}$ returns the set of both, as in (59).

$$
\begin{aligned}
& \llbracket \operatorname{ANS}_{F} \rrbracket((58))(\mathrm{w}) \\
& =\left\{\begin{array}{l}
\lambda \mathrm{w}: \diamond_{\mathrm{w}} \exists ! \mathrm{y}[\operatorname{letter}(\mathrm{y}) \wedge \operatorname{add}(\mathrm{y})] \cdot \diamond_{\mathrm{w}}(\operatorname{letter}(a) \wedge \operatorname{add}(a)), \\
\lambda \mathrm{w}: \diamond_{\mathrm{w}} \exists ! \mathrm{y}[\operatorname{letter}(\mathrm{y}) \wedge \operatorname{add}(\mathrm{y})] \cdot \diamond_{\mathrm{w}}(\operatorname{letter}(r) \wedge \operatorname{add}(r))
\end{array}\right\}
\end{aligned}
$$

In this way, given the Hamblin set in (58), (57) is predicted to have a mentionsome reading, where any true proposition in the Hamblin set counts as a complete answer. Dayal (2016) suggests that modalized singular which questions do not allow for mention-some. When low uniqueness is supported, however, a mention-some reading does emerge, as we predict. ${ }^{6}$

Suppose that Mary is playing a game, where she is shown a word skeleton, and will win a prize if she can make any word from the skeleton. Before the game, she is told that there might be more than one way to make a word in some rounds. In one round, the skeleton $f o \_m$ appears. It then seems felicitous for Mary to ask (57) as a mention-some question, asking the responder to name some letter that could be added to make a word. Consistent with the ANS-set in (59), the responder could completely resolve the question by answering either (60a) or (60b).
a. You could add $a$.
(complete answer)
b. You could add $r$.
(complete answer)

6 For a related observation, due to Roger Schwarzschild, see Dayal 2016: p. 75, fn. 19. 
In sum, $\mathrm{ANS}_{F}$, with variable scope uniqueness from which, correctly predicts both that basic singular which-questions should disallow mention-some, and that low uniqueness modalized questions should allow mention-some. In the former case, but not the latter, the projected uniqueness presupposition renders elements of the Hamblin set mutually exclusive. ${ }^{7}$

\section{Conclusion}

We have presented a localist analysis of the uniqueness presupposition of singular wh-questions according to which the presupposition is not triggered by ANS, as in Dayal's (1996) globalist analysis, but by an operator within the question nucleus, such as the $w h$ itself. The analysis is motivated by low uniqueness readings, which we attributed to reconstruction of the $w h$-phrase to a narrow scope site.

A number of issues remain open. First, constraints on the distribution of low uniqueness readings merit study. Can uniqueness be introduced beneath operators other than existential teleological and epistemic modals? If uniqueness comes from the $w h$-item, constraints on low uniqueness should, all things equal, track constraints on narrow scope for the wh-phrase. Moreover, multiple singular which-questions merit study in light of our conclusions. Which student saw which professor? does not presuppose that exactly one student saw exactly one professor, but rather that each student saw exactly one professor. How is that derived if uniqueness is locally triggered? We hope to take up these questions in future work.

7 While we have focused on mention-some, low uniqueness questions also seem to allow mention-all readings. These are perhaps clearest with epistemic modals. Suppose that the rules of Mary's game are changed so that there is a particular word hidden under the skeleton, and Mary must guess the right word to win. It then seems natural to ask (A) with the intention of soliciting all letters that could be missing, as in (B), so Mary can entertain all options before guessing the word.

$$
\begin{aligned}
& \text { A: Which letter could be missing in } f o \_m \text { ? } \\
& \text { B: } \quad A \text { or } r .
\end{aligned}
$$

To derive mention-all, low uniqueness questions must have a parse where which takes scope below the modal, but the Hamblin set is closed under conjunction, unlike in Section 4. One possibility, suggested in Xiang 2019, is that mention-all in our low uniqueness cases derives from a parse like the one in fn. 1. Adapting Xiang's proposal, ? (we assume optionally) quantifies over quantifiers and leaves a quantifier-type trace under could, as in (ii). The domain of quantification includes, for example, the Montague Lift of $a$ and $r$ and the disjunctive quantifier $a$ or $r$. A covert operator achieves free choice strengthening in the disjunctive answer from $\diamond(\operatorname{miss}(a) \vee \operatorname{miss}(r))$ to $(\diamond(\operatorname{miss}(a))$ $\wedge \diamond(\operatorname{miss}(r)))$, yielding the mention-all answer. This derivation captures the fact that, in (i), the mention-all answer is naturally expressed with a free choice disjunction.

$$
\text { [CP ? } \lambda 2 \text { [EXH [TP could [ } \mathrm{t}_{2 ; e s t, s t} \lambda 1{ }_{{ }_{v} P} \text { [which } \mathrm{t}_{1 ; e} \text { letter] be missing]]]]] }
$$


Hirsch and Schwarz

\section{References}

Champollion, Lucas, Ivano Ciardelli \& Floris Roelofsen. 2017. On questions and presuppositions in typed inquisitive semantics. Handout for talk at 2nd Workshop on Inquisitiveness Below and Beyond the Sentence Boundary (InqBnB), University of Amsterdam.

Dayal, Veneeta. 1996. Locality in wh quantification. Dordrecht: Kluwer.

Dayal, Veneeta. 2016. Questions. Oxford: Oxford University Press.

Fox, Danny. 2013. Mention some readings. Lecture notes, Massachusetts Institute of Technology.

Fox, Danny. 2018. Partition by exhaustification: Comments on Dayal 1996. In Uli Sauerland \& Stephanie Solt (eds.), Sinn und Bedeutung 22, vol. 2 ZASPiL 61, 403-434. Berlin: Leibniz-Centre General Linguistics.

George, B. R. 2011. Question embedding and the semantics of answers: University of California, Los Angeles $\mathrm{PhD}$ dissertation.

Groenendijk, Jeroen \& Martin Stokhof. 1984. Studies on the semantics of questions and the pragmatics of answers: University of Amsterdam $\mathrm{PhD}$ dissertation.

Hackl, Martin. 2000. Comparative quantifiers: Massachusetts Institute of Technology $\mathrm{PhD}$ dissertation.

Higginbotham, James \& Robert May. 1981. Questions, quantifiers and crossing. The Linguistic Review 1(1). 41-80.

Link, Godehard. 1983. The logical analysis of plurals and mass terms: A latticetheoretical approach. In Reiner Bäuerle, Christoph Schwarze \& Arnim von Stechow (eds.), Meaning, Use, and the Interpretation of Language, 302-323. Berlin: de Gruyter.

Rullmann, Hotze. 1995. Maximality in the semantics of wh-constructions: University of Massachusetts Amherst PhD dissertation.

Rullmann, Hotze \& Sigrid Beck. 1998. Presupposition projection and the interpretation of 'which'-questions. In Devon Strolovitch \& Aaron Lawson (eds.), Semantics and Linguistic Theory (SALT) 8, vol. 8, 215-232.

Sharvy, Richard. 1980. A more general theory of definite descriptions. The Philosophical Review 607-624.

Spector, B. 2008. An unnoticed reading for wh-questions: Elided answers and weak islands. Linguistic Inquiry 39. 677-686.

Uegaki, Wataru. 2018. On the projection of the presupposition of embedded questions. In Katherine Blake, Forrest Davis, Sireemas Maspong \& Brynhildur Stefánsdóttir (eds.), Semantics and Linguistic Theory (SALT) 28, 789-808. Linguistic Society of America.

Uegaki, Wataru. 2019. The existential/uniqueness presupposition of whcomplements projects from the answers. manuscript, University of Edinburgh. 
Xiang, Yimei. 2016. Interpreting questions with non-exhaustive answers: Harvard University $\mathrm{PhD}$ dissertation.

Xiang, Yimei. 2019. Higher-order readings of $w h$ questions. Paper presented at University of Göttingen.

Xiang, Yimei \& Alexandre Cremers. 2017. Mentions-some readings of pluralmarked questions: Experimental evidence. In Andrew Lamont \& Katerina Tetzloff (eds.), North East Linguistics Society (NELS) 47 3, 261-274. University of Massachusetts, Amherst.

Aron Hirsch

Language, Logic, and Cognition Center.

The Hebrew University of Jerusalem

Jerusalem 91905

Israel

aronh@alum.mit.edu
Bernhard Schwarz

1085 Dr. Penfield Ave.

Department of Linguistics

McGill University

Montreal, QC H3A1A7

Canada

bernhard.schwarz@mcgill.ca 